\title{
Renal replacement therapy is an alarm sign of survival outcome in pediatric liver transplantation
}

\author{
Byung Min Yoo ${ }^{1}$, Su young Hong ${ }^{2}$, Suk Kyun Hong ${ }^{2}$, Yo Han Ahn ${ }^{3}$, Hee Kyung Kang ${ }^{3}$, Namjoon $\mathrm{Yi}^{2}$, Sanggyun Suh², \\ Eui Soo Han ${ }^{2}$, Kwang-Woong Lee ${ }^{2}$, Kyung-Suk Suh ${ }^{2}$
}

${ }^{1}$ Department of Surgery, Seoul National University College of Medicine, Seoul, Korea
${ }^{2}$ Department of Surgery, Seoul National University Hospital, Seoul, Korea
${ }^{3}$ Department of Pediatrics-Nephrology, Seoul National University Hospital, Seoul, Korea

Background: Research regarding the impact of renal replacement therapy (RRT) on long-term survival outcome in pediatric liver recipients are scarce.

Methods: Two hundred twenty-four patients under 18 years of age who received liver transplantation (LT) in Seoul National University Hospital were enrolled. Patients were divided into two groups: patients who underwent RRT (group R) and the others (group N). Primary end point was post-transplant patients' and grafts' survival outcome.

Results: Twenty-five patients (11.2\%) received perioperative or sequential RRT, including nine patients (36.0\%) who underwent kidney transplantation eventually. The most common indication of RRT was metabolic liver disease (44\%) followed by hepatorenal syndrome (40\%). RRT was initiated on preoperative period (48\%) and postoperative period (early postoperative period [20\%] and long-term follow-up period [32\%]). In group R, age at the point of LT (71.6 months vs. 19.1 months) was older and pediatric end-stage liver disease (PELD) score was lower (9.9 vs. 21.2), post-transplant hospital stay day (41 days vs. 27 days) was longer and the rate of hepatic artery thrombosis $(8 \%$ vs. $3.5 \%)$ was higher than group $N(P<0.05)$. Compared with group $N$, group $R$ had significantly lower patients' $(60 \%$ vs. $93 \%, \mathrm{P}<0.001)$ and grafts' survival rates $(68 \%$ vs. $93 \%, \mathrm{P}<0.001)$. Post-transplant RRT was one of the risk factors of patients' survival outcome as well as non-biliary atresia patients, hepatic artery thrombosis and re-transplantation. However, preoperative RRT was not a risk factor of survival outcome.

Conclusions: Post-transplant survival outcomes of children requiring RRT were significantly worse than children without RRT. Physicians should pay more attention to patients requiring post-LT RRT although they are older and have lower PELD score. 